\title{
Etiological Classifications of Transient Ischemic Attacks: Subtype Classification by TOAST, CCS and ASCO - A Pilot Study
}

\author{
Margareth Amort Felix Fluri Florian Weisskopf Henrik Gensicke Leo H. Bonati \\ Philippe A. Lyrer Stefan T. Engelter
}

Stroke Unit and Department of Neurology, University Hospital Basel, Basel, Switzerland

\author{
Key Words \\ Etiological classification - Transient ischemic attack • \\ Trial of ORG 10172 in Acute Stroke Treatment · Causative \\ Classification System - Atherosclerosis Small Vessel Disease \\ Cardiac Source Other Cause
}

\begin{abstract}
Background: In patients with transient ischemic attacks (TIA), etiological classification systems are not well studied. The Trial of ORG 10172 in Acute Stroke Treatment (TOAST), the Causative Classification System (CCS), and the Atherosclerosis Small Vessel Disease Cardiac Source Other Cause (ASCO) classification may be useful to determine the underlying etiology. We aimed at testing the feasibility of each of the 3 systems. Furthermore, we studied and compared their prognostic usefulness. Methods: In a single-center TIA registry prospectively ascertained over 2 years, we applied 3 etiological classification systems. We compared the distribution of underlying etiologies, the rates of patients with determined versus undetermined etiology, and studied whether etiological subtyping distinguished TIA patients with versus without subsequent stroke or TIA within 3 months. Results: The 3 systems were applicable in all 248 patients. A determined etiology with the highest level of causality was
\end{abstract}

assigned similarly often with TOAST (35.9\%), CCS (34.3\%), and ASCO (38.7\%). However, the frequency of undetermined causes differed significantly between the classification systems and was lowest for ASCO (TOAST: 46.4\%; CCS: 37.5\%; ASCO: $18.5 \%$; $p<0.001$ ). In TOAST, CCS, and ASCO, cardioembolism (19.4/14.5/18.5\%) was the most common etiology, followed by atherosclerosis (11.7/12.9/14.5\%). At 3 months, 33 patients (13.3\%, 95\% confidence interval 9.3-18.2\%) had recurrent cerebral ischemic events. These were strokes in 13 patients (5.2\%; 95\% confidence interval 2.8-8.8\%) and TIAs in 20 patients (8.1\%, 95\% confidence interval 5.0-12.2\%). Patients with a determined etiology (high level of causality) had higher rates of subsequent strokes than those without a determined etiology [TOAST: $6.7 \%$ (95\% confidence interval $2.5-14.1 \%)$ vs. $4.4 \%$ (95\% confidence interval $1.8-8.9 \%)$; CSS: 9.3\% (95\% confidence interval $4.1-17.5 \%$ ) vs. 3.1\% (95\% confidence interval 1.0-7.1\%); ASCO: 9.4\% (95\% confidence interval $4.4-17.1 \%$ ) vs. $2.6 \%$ (95\% confidence interval $0.7-6.6 \%)$ ]. However, this difference was only significant in the ASCO classification $(p=0.036)$. Using ASCO, there was neither an increase in risk of subsequent stroke among patients with incomplete diagnostic workup (at least one subtype scored 9) compared with patients with adequate workup (no subtype scored 9), nor among patients with multiple causes compared with patients with a single cause. Conclusion: In

\section{KARGER \\ Fax +4161306 1234 \\ E-Mail karger@karger.ch}

www.karger.com
C) 2012 S. Karger AG, Basel

1015-9770/12/0336-0508\$38.00/0

Accessible online at:

www.karger.com/ced
Margareth Amort, MD

Department of Neurology and Stroke Unit, University Hospital Basel

Petersgraben 4

$\mathrm{CH}-4031$ Basel (Switzerland)

Tel. +41 61265 2525, E-Mail mamort@uhbs.ch 
TIA patients, all etiological classification systems provided a similar distribution of underlying etiologies. The increase in stroke risk in TIA patients with determined versus undetermined etiology was most evident using the ASCO classification.

Copyright $\odot 2012$ S. Karger AG, Basel

\section{Introduction}

The risk of subsequent stroke after a transient ischemic attack (TIA) ranges from 9 to $20 \%$ at 90 days [1-8]. For ischemic stroke, the criteria validated for the Trial of ORG 10172 in Acute Stroke Treatment (TOAST) [9] provide an established system to classify the underlying etiology. More recently, the SSS-TOAST (Stop Stroke Study TOAST system) [10] with the Causative Classification System (CCS) [11], a computerized web-based algorithm (http://ccs.martinos.org), based on available evidence for the association of stroke with risk markers and the ASCO classification of stroke (A for atherosclerosis, $\mathrm{S}$ for small vessel disease, $\mathrm{C}$ for cardiac source, $\mathrm{O}$ for other causes) [12], have been introduced in order to improve etiological assignment and to reduce the proportion of patients classified as having an undetermined cause [13]. In comparison with TOAST and CCS, the ASCO classification combines clinical data relevant for 4 stroke mechanisms in a single code for individual patients.

In TIA, evidence on the usefulness of etiological classification systems is sparse. The TOAST criteria have been applied in patients with TIA [5]. However, a major limitation of the TOAST classification is the large proportion of patients labeled as 'undetermined etiology' [13]. The more recent CCS and ASCO classifications have not been evaluated in TIA patients. In particular, it is unknown whether the newer classification systems result in a lower rate of patients with 'undetermined etiology'. Etiology is an important predictor of the risk of subsequent stroke after TIA, as shown in studies using the TOAST classification [3]. Whether the newer classification systems (i.e. the CCS and the ASCO system) are superior to TOAST in identifying patients at high risk for subsequent stroke is unclear.

The Basel Stroke Unit program includes a prospective registry and defines management pathways. In summer 2006, we designed a prospective single-center TIA study with the following objectives: firstly, to evaluate the quality of the management of TIA patients in our hospital [14] and to test whether a refinement of the ABCD score increases the predictability of recurrent ischemic events
[15]; secondly, to explore features which are best suited to distinguish between TIA and disorders mimicking TIA [16], and thirdly to evaluate the usefulness of etiological classification systems in patients with TIA. Our objectives for the present study were first, to compare the distribution of underlying etiologies between classification systems; second, to investigate whether the use of CCS and ASCO classifications increase the proportion of TIA patients with 'determined etiology' compared with the TOAST classification, and third, to test whether CCS and ASCO are superior to TOAST in identifying patients at high risk for recurrent stroke.

\section{Methods}

\section{Setting and Study Objectives}

Over a period of 48 months (November 5th, 2006 until November 4th, 2008), all consecutive patients admitted to the emergency department with the putative diagnosis of TIA, established by the admitting physician at the emergency department, were screened. For the current study, we selected all patients with a final diagnosis of TIA according to the NINDS criteria [17], based on the consensus opinion of two stroke neurologists, adopting an approach similar to that used for distinguishing stroke from mimic [18]. Adhering to the classical definition, a TIA was defined as an acute loss of focal cerebral or ocular function with symptoms lasting $<24 \mathrm{~h}$, that after adequate investigations was presumed to be due to embolic or thrombotic vascular disease.

Data were collected using a standardized case report form which was designed prior to the study. This form was prospectively used in all patients. The following variables were systematically assessed: demographic variables, clinical symptoms, risk factor profile, based on established criteria as used in previous studies [19], ABCD and $\mathrm{ABCD}^{2}$ scores, blood pressure values, and findings of etiological investigations (electrocardiogram, ECG; transthoracic/transesophageal echocardiography, TTE, TEE), neuroimaging results, and stroke prevention treatments.

Appropriate measures of stroke prevention were initiated immediately based on current guidelines $[20,21]$. This included the start of antithrombotic agents, statins, antihypertensive therapy and lifestyle counseling, as well as specific interventions tailored to the underlying etiology, based on the TOAST criteria. According to our in-house TIA guidelines [17], patients with an ABCD score of $\leq 4$ points [6], no acute ischemic lesions on diffusionweighted MR imaging (DWI), $\leq 50 \%$ stenosis of the extracranial arteries, and no high-risk abnormality on ECG were managed as outpatients. Patients with an ABCD score of 5 or more points or fulfilling at least one of the other aforementioned criteria were hospitalized.

Considering all available data, a single stroke physician assigned all patients to an etiological subtype using the TOAST, CCS and ASCO classification based on published criteria [9-11, 22] as summarized below. 


\section{Classifications}

Trial of Org 10172 in Acute Stroke Treatment

The etiological diagnosis according to the criteria of the TOAST [9] is based on clinical features, neuroimaging findings, and the results of diagnostic studies. 'Probable' and 'possible' diagnoses are made based on the physician's certainty of diagnosis. A 'probable' diagnosis requires that all findings after a complete workup be consistent with one subtype, and other etiologies have been excluded. A 'possible' diagnosis is made when available data suggest a specific subtype, even if some paraclinical examinations have not been performed [9]. The diagnosis of a probable subtype in TOAST in our study was only made if all of the following studies were performed: cerebral MRI including DWI, MR or computed tomography (CT) angiography, Doppler and duplex sonography alone or in combination, TTE, TEE, 12-lead ECG, and hemogram including platelet count and prothrombin time.

The TOAST classification denotes 5 subtypes of ischemic stroke: (1) large artery atherosclerosis (LAA), (2) cardioembolism (CE), (3) small vessel occlusion (SVO), (4) stroke of other determined etiology, and (5) stroke of undetermined etiology with the subcategories: two or more causes identified, negative evaluation and incomplete evaluation [9]. We applied the TOAST classification as previously described [9].

\section{Causative Classification System}

To weigh coexisting pathological findings in stroke patients, the SSS-TOAST classifies each of the 5 TOAST subtypes as either 'evident', 'probable', or 'possible' as an expression of the strength of association between clinical and imaging features with particular stroke mechanisms [10].

The SSS-TOAST was designed to improve the interexaminer reliability of the TOAST system and to minimize the proportion of stroke of undetermined etiology by eliminating the subcategory 'two or more causes identified' (these patients are assigned to a specific etiology based on the presence of specific characteristics of the stroke that make one mechanism more probable than others), and subdivides 'negative evaluation' in cryptogenic embolism and other cryptogenic stroke. We used the CCS [11], a computerized web-based algorithm of SSS-TOAST allowing a rapid classification of our TIA cohort (http://ccs.martinos.org).

\section{ASCO Classification}

The ASCO classification categorizes strokes along the following 4 causes: atherosclerosis (A), small vessel disease (S), cardiac source $(\mathrm{C})$, and other cause $(\mathrm{O})$. The presence and causal relationship to the stroke is graded for each potential cause according to the following categories: 1 , for definitely a potential cause of the index stroke; 2 , if causality is uncertain; 3 , if disease is present, but unlikely a direct cause of the index stroke; 0 for absent disease, and 9 , for insufficient workup [12].

\section{Comparison of the Classifications}

First, we compared the proportions of patients assigned to the highest level of causality of an underlying cause (i.e. TOAST: all cases with probable etiology; CCS: all cases with evident etiology; ASCO: all cases with a causality score of 1 for at least one etiology); the proportions of patients with high or moderate levels of causality (TOAST: all cases with probable or possible etiology; CCS: all cases with evident, probable and possible etiology; ASCO: all cases with a causality score of 1 or 2 for at least one etiology), and the proportions of patients with undetermined cause (TOAST: all cases classified as undetermined etiology; CCS: all cases assigned to undetermined causes and unclassified; ASCO: all cases without a causality score of 1 or 2 ) between the three classifications.

Second, we investigated the usefulness of the different classification systems in estimating the risk of subsequent stroke. In particular, we compared the proportion of patients with any stroke occurring within 3 months of the index TIA (a) between patients with the highest level of etiological causality and those without determined etiology in each classification, the influence of TIA mechanism on recurrence of stroke, and (b) between patients with more than one etiology which might have caused the TIA (TOAST: undetermined etiology due to more than one possible cause; CSS: unclassified; ASCO: more than one etiology with causality score of 1) and the remaining patients. In addition, 3-month stroke risk was compared (c) between patients with incomplete and those with complete diagnostic workup for their TIA; (d) between those managed as inpatients and those managed as outpatients, and (e) between patients with and without DWI lesions in the initial MRI. We calculated the frequency of the TIA mechanisms in each classification and their influence on recurrent stroke.

The primary outcome event for this study was the occurrence of any stroke within 90 days of the initial TIA. The secondary outcome event was the combination of any stroke or recurrent TIA within this period. Three-month outcome was assessed in clinical visits by stroke neurologists, who were unaware of the etiology of the initial TIA. If patients refused a follow-up visit, a telephone interview was performed using a standardized questionnaire to assess signs or symptoms of any vascular events. In case this assessment was not entirely normal, an urgent clinical visit (with further investigations available) by a stroke neurologist was scheduled. All outcome events were adjudicated by a senior stroke neurologist as also done in a previous study [15].

\section{Statistical Analysis}

Data are given as mean and standard deviation and odds ratios with $95 \%$ confidence intervals (CI), unless stated otherwise. Fisher's exact test was used to compare the proportion of patients with etiological assignments of high and high or moderate causality, as well as the proportion of patients with subsequent stroke between different patient groups, as defined above. Analyses were performed using SPSS 15.0 (Chicago, Ill., USA).

\section{Ethics}

The local ethics committee approved the study (No. 234/08).

\section{Results}

\section{Study Population}

The study population comprised 248 patients. Study patients had a mean age of 69.4 years; $60 \%$ were male. One hundred and three $(41.5 \%)$ patients were hospitalized according to the criteria defined above. The other patients were managed in an ambulatory setting. The 3 classifica- 
tion systems were applicable in all patients. The assignment was based on the clinical assessment (available in 248 patients; $100 \%$ ), results of CT with CT angiography (156; 62.9\%), MRI including DWI and MR angiography (203; 81.9\%), TTE (164; 66.1\%), TEE $(18 ; 7.3 \%)$ and neurosonology $(233 ; 94 \%)$, ECG $(248 ; 100 \%)$, and 24 -hour ECG $(\mathrm{n}=112 ; 45.2 \%)$. Hyperintense DWI lesions were found in 77 patients (31\%). Patient characteristics at baseline are shown in table 1 .

At 3 months, 33 patients $(13.3 \%$, 95\% CI 9.3-18.2\%) had recurrent cerebral ischemic events. These were strokes in 13 patients $(5.2 \%$; $95 \%$ CI $2.8-8.8 \%)$ and TIAs in 20 patients $(8.1 \%, 95 \%$ CI $5.0-12.2 \%)$. In 14 patients (5.6\%), events occurred within 48 hours, in 7 patients between $>48 \mathrm{~h}$ and $\leq 7$ days, in 10 patients between 8 and 30 days, and in 2 patients between 31 and 90 days.

\section{Causality and Etiology}

The highest level of etiological causality was assigned similarly often with the TOAST $(35.9 \% ; n=89)$, the CCS $(34.3 \% ; \mathrm{n}=85)$, and the ASCO classification $(38.7 \% ; \mathrm{n}=$ 96). The proportion of patients assigned a high or moderate level of causality did not differ significantly between classifications, i.e., TOAST: $53.6 \%(n=133)$, CCS: 62.5\% ( $\mathrm{n}=155)$, and ASCO: $55.6 \%(\mathrm{n}=138)$. The frequency of undetermined causes differed significantly between the classification systems and was lowest for ASCO (TOAST: 46.4\%; CCS: 37.5\%; ASCO: 18.5\%; p < 0.001 ) (table 2). One hundred and fifteen TIA patients (46.4\%) were classified as having an undetermined cause by TOAST. The etiology remained unknown in 45 patients (18.1\%) despite adequate evaluation, in 13 (5.2\%) more than one possible cause was present, and in 57 (23.0\%) the evaluation was not complete. The CCS classification identified 93 (37.5\%) patients without determined etiology: unknown cryptogenic embolism, 3 patients (1.2\%); unknown other cryptogenic, 31 patients (12.5\%); incomplete evaluation, 53 patients (21.4\%), and unclassified causes, 6 patients (2.4\%).

In 28 of the 115 TIA patients classified by TOAST as having an undetermined etiology, the ASCO classification revealed a determined etiology. This included LAA in 15 patients ( $\mathrm{A} 1, \mathrm{n}=3,2.6 \% ; \mathrm{A} 2, \mathrm{n}=12,10.4 \%)$, SVO in $9(\mathrm{~S} 1, \mathrm{n}=3,2.6 \%$; $\mathrm{S} 2, \mathrm{n}=6,5.2 \%), \mathrm{CE}$ in $11(\mathrm{C} 1, \mathrm{n}=9$, $7.8 \% ; \mathrm{C} 2, \mathrm{n}=2,1.7 \%)$, and other causes in 2 patients $(\mathrm{O} 1$, $\mathrm{n}=1,0.9 \% ; \mathrm{O} 2, \mathrm{n}=0,0 \%)$.

$\mathrm{CE}$ was the most frequent determined etiology in all classifications (TOAST probable, 19.4\%; CCS evident, $14.5 \%$; ASCO 1, 18.5\%), followed by LAA (TOAST probable, 11.7\%; CSS evident, 12.9\%; ASCO 1, 14.5\%), SVO
Table 1. Baseline characteristics in patients with TIA $(\mathrm{n}=248)$

$\begin{array}{lc}\text { Demographic data } & \\ \text { Mean age, years } & 69.4 \pm 13 \\ \text { Male gender } & 149(60.1) \\ \text { Vascular risk factors } & 175(70.6) \\ \text { Hypertension } & 47(19.0) \\ \text { Coronary heart disease } & 53(21.4) \\ \text { Current smoking } & 41(16.5) \\ \text { Diabetes mellitus } & 96(38.7) \\ \text { Hypercholesterolemia } & 54(21.8) \\ \text { Family history of stroke } & 22(8.9) \\ \text { Peripheral arterial disease } & 37(14.9) \\ \text { Atrial fibrillation } & \end{array}$

\begin{tabular}{lc}
\hline Secondary preventive drugs & \\
Antithrombotics & $153(61.7)$ \\
ASS & $68(27.4)$ \\
Clopidogrel & $41(16.5)$ \\
Phenprocoumon & $10(4.0)$ \\
Heparin & \\
Antihypertensive drugs & $66(26.6)$ \\
Diuretics & $80(32.7)$ \\
ACE inhibitor & $51(20.6)$ \\
AT II inhibitor & $38(15.3)$ \\
Calcium antagonist & $87(35.1)$ \\
Beta-blockers & $154(62.1)$ \\
Statins & $21(8.5)$ \\
OAD & $8(3.2)$ \\
Insulin & $24.4 \pm 6.9$ \\
Mean BMI & $4.06 \pm 1.34$ \\
Mean ABCD score & $4.23 \pm 1.4$ \\
Mean ABCD2 score &
\end{tabular}

Figures are mean $\pm \mathrm{SD}$ or $\mathrm{n}(\%)$.

$\mathrm{OAD}=$ Oral antidiabetics; $\mathrm{ABCD}$ and $\mathrm{ABCD} 2=$ prognostic scores developed to determine stroke risk after TIA [27].

${ }^{1}$ Based on $201 / 248$ patients.

(TOAST probable, 4.4\%; CCS evident, 4.4\%; ASCO 1, $6 \%$ ), and other causes (TOAST probable, $0.4 \%$; CCS evident, 2.4\%; ASCO 1, 2.4\%). Details are shown in table 2.

The numbers of etiological assignments which were concordant and discordant between classifications are displayed in figure 1. LAA was concordantly assigned in all 3 classifications to 17 of the 47 patients (36\%), in whom LAA was the cause in at least one classification. For CE, this proportion was 22 of 71 patients (31\%), for SVO 6 of 20 patients (30\%) and for other causes 0 of 8 patients. There was no clear pattern of discordance across the classification systems.

Applying all scoring systems, patients with assignment to the highest level of causality (TOAST probable, 
Table 2. Etiological classification of TIA patients $(n=248)$ with TOAST, CCS and ASCO classification

\begin{tabular}{|c|c|}
\hline \multicolumn{2}{|l|}{ TIA patients } \\
\hline TOAST, probable or possible etiology & $133(53.6)$ \\
\hline Probable etiology & $89(35.9)$ \\
\hline Possible etiology & $44(17.7)$ \\
\hline TOAST, large artery disease & $46(19.4)$ \\
\hline Probable & $29(63)$ \\
\hline Possible & $17(37)$ \\
\hline TOAST, cardioembolic & $66(26.6)$ \\
\hline Probable & $48(72.7)$ \\
\hline Possible & $18(27.3)$ \\
\hline TOAST, small vessel occlusion & $16(6.5)$ \\
\hline Probable & $11(68.8)$ \\
\hline Possible & $5(31.3)$ \\
\hline TOAST, other determined etiology & $5(2.0)$ \\
\hline Probable & $1(20)$ \\
\hline Possible & $4(80)$ \\
\hline TOAST, undetermined etiology & $115(46.4)$ \\
\hline TOAST, evaluation negative & $45(18.1)$ \\
\hline TOAST, more than one possible cause & $13(5.2)$ \\
\hline TOAST, evaluation not complete & $57(23.0)$ \\
\hline CCS, evident, probable or possible etiology & $155(62.5)$ \\
\hline Evident etiology & $85(34.3)$ \\
\hline Probable or possible etiology & $116(46.8)$ \\
\hline CCS all assigned to LAA & $63(25.4)$ \\
\hline CCS LAA evident & $32(12.9)$ \\
\hline CCS LAA probable & $6(2.4)$ \\
\hline CCS LAA possible & $25(10.1)$ \\
\hline CCS all assigned to cardioaortic embolism (CAE) & $68(27.4)$ \\
\hline CCS CAE evident & $36(14.5)$ \\
\hline CCS CAE probable & $2(0.8)$ \\
\hline CCS CAE possible & $30(12.1)$ \\
\hline CCS all assigned to SVO & $17(6.9)$ \\
\hline CCS SVO evident & $11(4.4)$ \\
\hline CCS SVO probable & $0(0)$ \\
\hline CCS SVO possible & $6(2.4)$ \\
\hline CCS all assigned to other causes (OC) & $7(2.8)$ \\
\hline CCS OC evident & $6(2.4)$ \\
\hline CCS OC probable & $1(0.4)$ \\
\hline CCS OC possible & $0(0)$ \\
\hline CCS all assigned to undetermined causes (UC) & $93(37.5)$ \\
\hline CCS unknown cryptogenic embolism & $3(1.2)$ \\
\hline CCS unknown other cryptogenic & $31(12.5)$ \\
\hline CCS incomplete evaluation & $53(21.4)$ \\
\hline CCS unclassified & $6(2.4)$ \\
\hline
\end{tabular}

\begin{tabular}{lc} 
TIA patients & \\
\hline ASCO, score of 1 or 2 & $138(55.6)$ \\
Score of 1 & $96(38.7)$ \\
Score of 2 & $52(21.1)$ \\
ASCO A0 & $58(23.4)$ \\
ASCO A1 & $36(14.5)$ \\
ASCO A2 & $32(12.9)$ \\
ASCO A3 & $99(39.9)$ \\
ASCO A9 & $23(9.3)$ \\
ASCO S0 & $87(35.1)$ \\
ASCO S1 & $15(6.0)$ \\
ASCO S2 & $12(4.8)$ \\
ASCO S3 & $95(38.3)$ \\
ASCO S9 & $39(15.7)$ \\
ASCO C0 & $69(27.8)$ \\
ASCO C1 & $46(18.5)$ \\
ASCO C2 & $10(4.0)$ \\
ASCO C3 & $38(15.3)$ \\
ASCO C9 & $85(34.3)$ \\
ASCO O0 & $185(74.6)$ \\
ASCO O1 & $6(2.4)$ \\
ASCO O2 & $1(0.4)$ \\
ASCO O3 & $11(4.4)$ \\
ASCO O9 & $45(18.1)$ \\
\hline
\end{tabular}

Figures in parentheses are percentages.

nificance only for the ASCO score $(\mathrm{p}=0.036)$. There was no subtype of etiology at a statistically higher risk of stroke recurrence, but a trend to higher recurrence in LAA classified in TOAST $(\mathrm{p}=0.07)$.

\section{More than One Etiology}

The presence of more than one etiology which may have caused the TIA, defined as 'more than one possible cause' ( $\mathrm{n}=13 / 248)$ in the TOAST classification, 'unclassified' in CCS, and more than one etiology with a causality score of $1(n=6 / 248)$ or a combination of scores of 1 and $2(\mathrm{n}=11 / 248)$ in the ASCO classification, did not increase the risk of stroke (TOAST $\mathrm{p}=0.51, \mathrm{CCS} \mathrm{p}=1.0$, ASCO $\mathrm{p}=0.58 / \mathrm{p}=1.0$ ) after 3 months (table 3 ).

\section{Incomplete Workup}

Incomplete evaluation in the ASCO classification (represented by a score of 9) exists only at the level of single etiologies. For the present study, incomplete workup was arbitrarily defined as the presence of more than one etiology labeled 9, which applied to 46 patients (18.5\%). 
Table 3. Association between etiological assignment in the single classifications with 3-month risk of stroke

\begin{tabular}{|c|c|c|c|c|}
\hline TOAST assigned to probable & $89 / 248(35.9)$ & $6 / 89(6.7)$ & $83 / 89(93.3)$ & 0.554 \\
\hline TOAST without determined etiology & $159 / 248(64.1)$ & $7 / 159(4.4)$ & $152 / 159(95.6)$ & 0.554 \\
\hline CCS, all assigned to evident & $86 / 248(34.7)$ & $8 / 86(9.3)$ & $78 / 86(90.7)$ & 0.068 \\
\hline ASCO never 1 & $152 / 248(61.3)$ & $4 / 152(2.6)$ & $148 / 152(97.4)$ & 0.036 \\
\hline TOAST, two or more causes & $13 / 248(5.2)$ & $1 / 13(7.7)$ & $12 / 13(92.3)$ & 0.512 \\
\hline CCS, unclassified & $6 / 248(2.4)$ & $0 / 6(0.0)$ & $6 / 6(100)$ & 1.0 \\
\hline ASCO two or more 1 & $6 / 248(2.4)$ & $1 / 6(16.7)$ & $5 / 6(83.3)$ & 0.279 \\
\hline ASCO at least one 9 & $105 / 248(42.3)$ & $2 / 105(1.9)$ & $103 / 105(98.1)$ & 0.48 \\
\hline
\end{tabular}

p values of Fisher's exact test. Figures in parentheses are percentages.

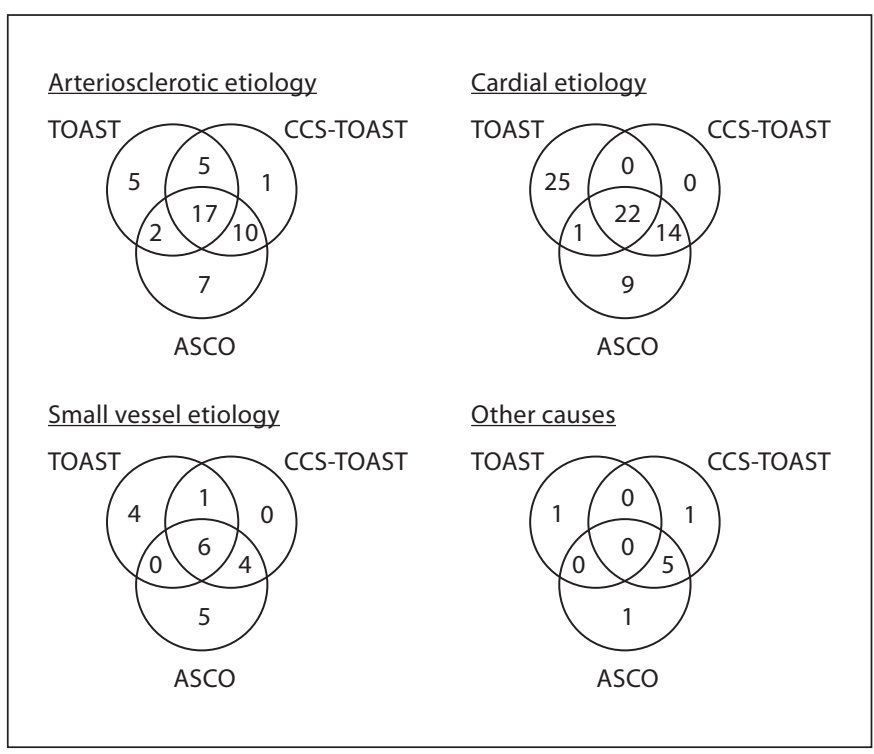

Fig. 1. Proportion of concordant subtyping for each etiological subtypes (considering the highest level of confidence for subtyping) displayed in Venn diagrams.

The proportion of patients with incomplete evaluation was similar in all classifications (TOAST $23.0 \%$, CCS 21.4\%, ASCO 18.5\%).

In all three classifications, the risk of stroke within 3 months of the index TIA did not differ significantly between patients who had incomplete and those with com- plete evaluation and adequate investigation (TOAST $\mathrm{p}=$ $0.74, \operatorname{CCS} \mathrm{p}=0.71$, ASCO $\mathrm{p}=0.09$ ).

The portion of study participants with incomplete evaluation was higher among those managed as outpatients than those who were hospitalized, in all classifications. Among all hospitalized patients $(\mathrm{n}=103)$ and all outpatients $(\mathrm{n}=145)$ the numbers of those with incomplete evaluations were $17(17 \%)$ and $40(28 \%)$ in the TOAST classification $(\mathrm{p}=0.047), 16(16 \%)$ and $37(26 \%)$ in CCS ( $\mathrm{p}=0.061)$, and $35(34 \%)$ and $70(48 \%)$ in ASCO $(\mathrm{p}=0.027)$, respectively.

The finding of a DWI lesion (31\%) correlated with a classification in high level of confidence in all three systems $(\mathrm{p}<0.001)$.

\section{Discussion}

In TIA patients - unlike in some stroke patients pathological confirmation of the underlying cause for the ischemic event is not feasible, because patients survive the episode [10]. Little information exists on classification systems in TIA patients, as all systems had been developed for stroke patients. TOAST had been developed for standardizing the etiological assignment in clinical trials about a novel therapy for acute ischemic stroke and has widely been used in stroke patients thereafter [9]. In contrast, there are only few studies using TOAST in TIA [5, 23], or combined TIA/stroke cohorts [24, 25]. To the best of our knowledge, the more recently developed CSS and 
ASCO classifications have never been tested in TIA patients so far. Thus, our study was the first time to compare the three classifications in TIA.

As key findings, first we did not observe relevant differences between the three classification systems in the proportion of patients in who causes with high or high-tomoderate levels of causality were identified. Second, the ASCO classification yielded a lower proportion of patients with undetermined cause than the TOAST and CSS classifications. Third, patients with determined etiologies had a higher risk of stroke within the 3 months following TIA than patients with an undetermined etiology. These findings stress the prognostic importance of the underlying etiology as shown in previous research [5,26]. Nevertheless, the latter finding is surprising, as all evidence-based treatment options were applied to prevent subsequent strokes in our cohort [17]. Thus, the fact that the frequency of strokes in the determined etiology group - according to ASCO - is about 3 times higher than that in the undetermined etiology group indicates the need for more efficacious means of stroke prevention in these patients.

Considering the classifications in a more theoretical way, there is an important difference in the relevance of confidence levels: the approach to attributing a found pathology to the cause of event. The confidence levels in TOAST and CCS were developed to describe the certainty of relation between the pathological finding and the event, while the confidence levels in ASCO are more a description of the abnormity. The theoretical advantage of the ASCO classification as a phenotypic classification system is to retain and describe information of all underlying diseases, so that a vascular risk profile of the patient is generated. These differences between the classification systems might be an explanation for the finding that only in the ASCO classification did patients with the highest level of causality have significantly higher rates of subsequent strokes.

Because of some fundamental differences in the definition of etiological subtypes between the classification systems, we avoided comparisons of single subtypes across the classification systems. An example is the distinct allocation of aortic arch plaques, which are assigned to arteriosclerosis in the ASCO classification and to 'cardioaortic embolism' in CSS. Probable LAA according to the TOAST classification was associated with a nonsignificant trend ( $\mathrm{p}=0.07)$ towards a higher risk of subsequent stroke, which is consistent with previous research [5]. None of the other etiologies in any classification were significantly associated with the occurrence of subsequent stroke.
In comparison to a recent study about subtyping in stroke patients [13], the TIA patients in our study more often had undetermined cases in all classifications: TOAST 46.4 versus $39.3 \%$, CSS 37.5 versus $26.2 \%$ and ASCO (no ASCO1 or ASCO2 evidence) 44.4 versus $42.3 \%$ in the aforementioned study. These differences are plausible because in TIA patients clinical data are usually restricted to reported symptoms, and the rate of transient focal neurological symptoms mimicking a cerebrovascular event ('TIA mimics') is higher than in stroke patients ('stroke mimics') [17]. More importantly, the distribution of etiologies based on the TOAST classification is similar to that of previous TIA cohorts, showing that our TIA patient cohort is comparable to others [5]. Despite adequate evaluation, the category 'undetermined etiology' is more often present in TIA patients analyzed by TOAST compared to recently analyzed stroke patients $[17.3 \%$ (current TIA study) versus 13.9\% (stroke study)] [13]. Likewise, the percentage of patients with incomplete evaluation was higher in TIA than in stroke patients [i.e., $23.0 \%$ (current TIA study) versus $11.0 \%$ (stroke study)] [13]. The lower rate of hospitalization, the assumed shorter length of stay of TIA patients and the transient character of the disease compared to stroke patients might account for this difference. This assumption is supported by the observation that the proportion of incomplete evaluation was lower in TIA patients treated as outpatients than in those being hospitalized.

The presence of more than one potential etiology was lower in our TIA cohort than in a recently published stroke cohort (5.2 vs. 14.4\%) [13]. This might again be explained by the lower rate of complete diagnostic workup in TIA compared to stroke patients.

Neither the presence of more than one cause for the index TIA nor incomplete diagnostic workup was associated with the risk of subsequent stroke. However, this finding should be interpreted cautiously due to the small number of patients in these categories.

We are aware of the following limitations. First, the relatively small number of TIA patients limited the analysis of patients with competing mechanisms. Second, the etiological assignment in single classifications was not done blinded to the assignment in the other classifications. Third, a gold standard for etiological assignment does not exist. Fourth, the risk of subsequent stroke is not only determined by the etiological classification per se, but also by measures of secondary prevention which are taken as result of the diagnostic evaluation. Thus, although our findings suggest that ASCO might be superior to the other classification systems in identifying TIA 
patients at particularly high risk of stroke, our findings should be considered preliminary. Therefore, our findings require confirmation by future research before the clinical utility can be assessed.

In conclusion, the practical application of the TOAST, the CCS, and the ASCO classification in a TIA cohort is feasible and revealed comparable results. Patients with a determined etiology seem to carry an increased risk for a subsequent stroke, but it remains to be confirmed whether etiological classifications might aid in identifying high-risk patients.

\section{Acknowledgements}

The study was supported by research grants of the Swiss Heart Foundation and the Foundation of Health and Cardio-Neurovascular Research, Basel, Switzerland.

\section{Disclosure Statement}

There are no conflicts of interest.

\section{References}

1 Coutts SB, Eliasziw M, Hill MD, Scott JN, Subramaniam S, Buchan AM, Demchuk AM: An improved scoring system for identifying patients at high early risk of stroke and functional impairment after an acute transient ischemic attack or minor stroke. Int J Stroke 2008;3:3-10.

$\checkmark 2$ Eliasziw M, Kennedy J, Hill MD, Buchan AM, Barnett HJM: Early risk of stroke after a transient ischemic attack in patients with internal carotid artery disease. CMAJ 2004; 170:1105-1109.

- 3 Ois A, Gomis M, Rodríguez-Campello A, Cuadrado-Godia E, Jiménez-Conde J, PontSunyer C, Cuccurella G, Roquer J: Factors associated with a high risk of recurrence in patients with transient ischemic attack or minor stroke. Stroke 2008;39:1717-1721.

4 Kleindorfer D, Panagos P, Pancioli A, Khoury J, Kissela B, Woo D, Schneider A, Alwell K, Jauch E, Miller R, Moomaw C, Shukla R, Broderick JP: Incidence and short-term prognosis of transient ischemic attack in a population-based study. Stroke 2005;36: 720-723.

5 Purroy F, Montaner J, Molina CA, Delgado P, Ribo M, Alvarez-Sabín J: Patterns and predictors of early risk of recurrence after transient ischemic attack with respect to etiologic subtypes. Stroke 2007;38:3225-3229.

-6 Rothwell PM, Coull AJ, Silver LE, Fairhead JF, Giles MF, Lovelock CE, Redgrave JNE, Bull LM, Welch SJV, Cuthbertson FC, Binney LE, Gutnikov SA, Anslow P, Banning AP, Mant D, Mehta Z: Population-based study of event-rate, incidence, case fatality, and mortality for all acute vascular events in all arterial territories (Oxford Vascular Study). Lancet 2005;366:1773-1783.

-7 Johnston SC, Gress DR, Browner WS, Sidney S: Short-term prognosis after emergency department diagnosis of TIA. JAMA 2000;284: 2901-2906.

TIA Subtype Classification by TOAST, CSS and ASCO
8 Hill MD, Yiannakoulias N, Jeerakathil T, Tu JV, Svenson LW, Schopflocher DP: The high risk of stroke immediately after transient ischemic attack: a population-based study. Neurology 2004;62:2015-2020.

-9 Adams HP, Bendixen BH, Kappelle LJ, Biller J, Love BB, Gordon DL, Marsh EE: Classification of subtype of acute ischemic stroke. Definitions for use in a multicenter clinical trial. TOAST. Trial of Org 10172 in Acute Stroke Treatment. Stroke 1993;24: 35-41.

10 Ay H, Furie KL, Singhal A, Smith WS, Sorensen AG, Koroshetz WJ: An evidencebased causative classification system for acute ischemic stroke. Ann Neurol 2005;58: 688-697.

-11 Ay H, Benner T, Arsava EM, Furie KL, Singhal $A B$, Jensen $M B$, Ayata $C$, Towfighi $A$, Smith EE, Chong JY, Koroshetz WJ, Sorensen AG: A computerized algorithm for etiologic classification of ischemic stroke: the Causative Classification of Stroke System. Stroke 2007;38:2979-2984.

12 Amarenco P, Bogousslavsky J, Caplan LR, Donnan GA, Hennerici MG: New approach to stroke subtyping: the A-S-C-O (phenotypic) classification of stroke. Cerebrovasc Dis 2009;27:502-508.

13 Marnane M, Duggan CA, Sheehan OC, Merwick A, Hannon N, Curtin D, Harris D, Williams EB, Horgan G, Kyne L, McCormack PME, Duggan J, Moore A, Crispino-O'Connell G, Kelly PJ: Stroke subtype classification to mechanism-specific and undetermined categories by TOAST, A-S-C-O, and causative classification system: direct comparison in the North Dublin population stroke study. Stroke 2010;41:1579-1586.

14 Weisskopf F, Fluri F, Amort M, Katan M, Bingisser R, Engelter ST, Lyrer PA: Prozessanalyse des Abklärungs- und Behandlungskonzeptes für Patienten mit Transitorischer Ischämischer Attacke im Universitätsspital Basel. SANP 2010;161:166-172.
15 Engelter ST, Amort M, Jax F, Weisskopf F, Katan M, Burow A, Bonati LH, Hatz F, Wetzel SG, Fluri F, Lyrer PA: Optimizing the risk estimation after a transient ischaemic attack - The ABCDE $\oplus$ score. Eur J Neurol 2012;19:55-61.

16 Amort M, Fluri F, Schäfer J, Weisskopf F, Katan M, Burow A, Bucher HC, Bonati LH, Lyrer PA, Engelter ST: Transient ischemic attack versus transient ischemic attack mimics: frequency, clinical characteristics and outcome. Cerebrovasc Dis 2011;32:5764.

17 Special report from the National Institute of Neurological Disorders and Stroke: classification of cerebrovascular diseases III. Stroke 1990;21:637-676.

18 Hand PJ, Kwan J, Lindley RI, Dennis MS, Wardlaw JM: Distinguishing between stroke and mimic at the bedside: the Brain Attack Study. Stroke 2006;37:769-775.

19 Fluri F, Hatz F, Voss B, Lyrer PA, Engelter ST: Restenosis after carotid endarterectomy: significance of newly acquired risk factors. Eur J Neurol 2010;17:493-498.

20 Easton JD, Saver JL, Albers GW, Alberts MJ, Chaturvedi S, Feldmann E, Hatsukami TS, Higashida RT, Johnston SC, Kidwell CS, Lutsep HL, Miller E, Sacco RL: Definition and evaluation of transient ischemic attack: a scientific statement for healthcare professionals from the American Heart Association/ American Stroke Association Stroke Council; Council on Cardiovascular Surgery and Anesthesia; Council on Cardiovascular Radiology and Intervention; Council on Cardiovascular Nursing; and the Interdisciplinary Council on Peripheral Vascular Disease. The American Academy of Neurology affirms the value of this statement as an educational tool for neurologists. Stroke 2009; 40 : 2276-2293.

$-$

Cerebrovasc Dis 2012;33:508-516 
21 Adams HP, Del Zoppo G, Alberts MJ, Bhatt DL, Brass L, Furlan A, Grubb RL, Higashida RT, Jauch EC, Kidwell C, Lyden PD, Morgenstern LB, Qureshi AI, Rosenwasser RH, Scott PA, Wijdicks EFM, del Zoppo H: Guidelines for the early management of adults with ischemic stroke: a guideline from the American Heart Association/American Stroke Association Stroke Council, Clinical Cardiology Council, Cardiovascular Radiology and Intervention Council, and the Atherosclerotic Peripheral Vascular Disease and Quality of Care Outcomes in Research Interdisciplinary Working Groups: The American Academy of Neurology affirms the value of this guideline as an educational tool for neurologists. Circulation 2007;115:e478-e534.
22 Amarenco P, Bogousslavsky J, Caplan LR, Donnan GA, Hennerici MG: Classification of stroke subtypes. Cerebrovasc Dis 2009;27: 493-501.

23 Mlynash M, Olivot J, Tong DC, Lansberg MG, Eyngorn I, Kemp S, Moseley ME, Albers GW: Yield of combined perfusion and diffusion MR imaging in hemispheric TIA. Neurology 2009;72:1127-1133.

24 Clark JM, Renier SA: A community stroke study: factors influencing stroke awareness and hospital arrival time. J Stroke Cerebrovasc Dis 2001;10:274-278.
25 Poppert H, Sadikovic S, Sander K, Wolf O, Sander D: Embolic signals in unselected stroke patients: prevalence and diagnostic benefit. Stroke 2006;37:2039-2043.

26 Calvet D, Touzé E, Oppenheim C, Turc G, Meder J, Mas J: DWI lesions and TIA etiology improve the prediction of stroke after TIA. Stroke 2009;40:187-192.

27 Josephson SA, Sidney S, Pham TN, Bernstein AL, Johnston SC: Higher ABCD2 score predicts patients most likely to have true transient ischemic attack. Stroke 2008;39:30963098 . 